\title{
La délivrance de titres et certificats par les hôpitaux aurait besoin d'un (bon) coup de barre
}

\author{
James Ducharme, MD, CM
}

Nous vivons à une époque où l'information est supposée être accessible universellement. Aussi l'obligation de se soumettre à un long processus de reconnaissance chaque fois qu'un médecin fait une demande d'un nouveau permis provincial semble-t-elle anachronique. Pire encore, il lui faut passer à travers un processus de délivrance de titres et certificats coûteux et apparemment aléatoire dans chaque hôpital où il désire travailler. Comment un tel processus de délivrance de titres et certificats a-t-il pu survivre si longtemps «en vase clos»? Quand on compare la démarche avec celle dans d'autres domaines d'activité en soins de santé, par exemple, la multitude d'applications qui permettent un accès mobile à des renseignements médicaux, les dossiers, la formation, et les sites Web facilement accessibles des collèges provinciaux de médecins et de l'Association médicale canadienne, on constate que les processus de délivrance de titres et certificats et d'autorisation d'exercer sont nettement archaïques.

Les collèges provinciaux de médecins font des efforts pour améliorer leurs processus administratifs de permis d'exercice de la médecine. Certes, le processus est soumis à la Loi constitutionnelle, qui stipule que la délivrance des permis d'exercice de la médecine est de compétence provinciale, mais des lois récentes ont permis à la Fédération des ordres des médecins du Canada d'uniformiser les exigences en matière de délivrance des permis d'exercice de la médecine. Cette «norme nationale» permettra à un médecin qui remplit les conditions requises à l'obtention d'un permis sans restriction dans une province d'être assuré d'obtenir ce même permis dans n'importe quelle autre province (James Stratford, service d'inscription, Ordre des médecins et chirurgiens de l'Ontario, communication personnelle, 4 février 2011).

En outre, la Fédération, de même que Le Conseil médical du Canada, a mis sur pied une nouvelle organisation, le Registre de compétences des médecins du Canada. Ce Registre national constituera un dépôt de données sur les médecins et procédera à la délivrance de titres et certificats au nom des autorités provinciales compétentes. À l'avenir, il devrait donc être simple, pour un médecin qui passe d'une province à l'autre, d'obtenir rapidement tout nouveau permis nécessaire. Le Registre et la Fédération travaillent également à élaborer un processus national de demande en ligne à l'aide d'un formulaire commun, qui sera envoyé directement aux collèges provinciaux de médecins concernés.

La délivrance de titres et certificats par les hôpitaux, contrairement aux étapes actuellement envisagées d'autorisation d'exercer dans les provinces, reste coûteuse, et chaque établissement travaille en vase clos. Depuis que je me suis établi en Ontario, en 2006, j'ai obtenu des droits hospitaliers dans plus de 10 établissements. Pourtant, je dois faire mention de deux cas ayant le même processus de délivrance de titres et certificats. Tandis que, dans certains établissements, il m'a fallu moins de 30 minutes pour remplir les formulaires nécessaires, dans un autre, le processus de délivrance de titres et certificats a pris presque 15 heures. Aucun hôpital n'est disposé à accepter les renseignements d'un autre établissement à moins qu'il appartienne à la même régie régionale de santé. Fait surprenant, les médecins qui s'engagent à faire de la suppléance dans des services d'urgence par l'intermédiaire de Professions Santé Ontario n'ont pas à se

Département de médecine d'urgence, Université McMaster, Hamilton, ON.

Demandes: Dr James Ducharme, 6711 Mississauga Road, bureau 404, Mississauga, ON L5N 2W3; paindoc22000@yahoo.com.

L'article a été évalué par les pairs. 
soumettre à quelque processus de délivrance de titres et certificats que ce soit par les hôpitaux. Alors, comment se fait-il qu'une exigence aussi courante, et sans doute facile à uniformiser, puisse être si différente?

Il va de soi que les centres de santé doivent s'assurer que leur personnel est autorisé, assuré, et exempt de risque médical ou juridique. Cependant, il n'est pas clair pourquoi un hôpital exigerait des renseignements sur la délivrance de titres et certificats qu'aucun autre hôpital ne demande, ou pourquoi chaque hôpital doit avoir son propre processus. Une solution possible serait de conserver les renseignements essentiels dans une base de données centrale. De fait, une bonne partie de ces renseignements sera bientôt conservée dans le Registre. Un seul formulaire normalisé suffirait.

Il serait même plus utile de séparer les exigences relatives à la délivrance de titres et certificats du processus d'orientation, essentiel mais généralement négligé des médecins. Dans le cadre du processus de délivrance de titres et certificats, de nombreux hôpitaux exigent l'étude complète d'un ou plusieurs modules de formation. Ceux-ci sont généralement élaborés au niveau local, à grands frais, malgré l'existence de matériel similaire facilement accessible ailleurs, dans le Web. Par exemple, certains hôpitaux en Ontario exigent l'étude complète de leurs propres modules de lutte contre les infections et de formation sur l'hygiène des mains pour la délivrance de titres et certificats même s'il existe des ressources similaires dans le site Web de l'Ordre des médecins et chirurgiens de l'Ontario. Ce type de matériel didactique appartient davantage à un processus d'orientation, qui devrait être entrepris par tout le personnel, y compris par les médecins, une fois les droits hospitaliers accordés. Ce genre d'orientation pourrait cibler des domaines propres à chaque fonction hospitalière et être lié à un site central qui contiendrait des modules communs à tous les hôpitaux.

Le temps est venu de collaborer davantage. Il semblerait raisonnable que l'Institut canadien d'information sur la santé oblige les hôpitaux à s'entendre sur un processus uniforme de délivrance de titres et certificats. Il devrait être possible de constituer et d'entretenir une base de données nationale sur les médecins, et ceux-ci devraient avoir l'obligation de mettre régulièrement à jour les renseignements susceptibles de devenir périmés. Cela pourrait facilement s'intégrer au système centralisé de délivrance de permis d'exercice de la médecine, actuellement envisagé.

Ainsi, grâce à une base de données nationale, les hôpitaux pourraient télécharger depuis un site central tous les renseignements nécessaires. Au lieu d'avoir un service de délivrance de titres et certificats par hôpital, il pourrait y en avoir un seul dans tout le pays. Le site permettrait de s'assurer que tous les médecins gardent leurs renseignements à jour, à défaut de quoi ils ne pourraient obtenir ou renouveler leurs droits hospitaliers. Les collèges provinciaux de médecins pourraient télécharger tout document de nature délicate, comme des plaintes pendantes ou réglées, et L'Association canadienne de protection médicale pourrait télécharger toute donnée pertinente exigée par les hôpitaux. Tous les renseignements confidentiels pourraient être protégés, et d'ailleurs ils le seraient certainement plus que dans des classeurs, à l'hôpital.

Il est plus que temps que les processus administratifs médicaux s'adaptent à l'époque où nous vivons. Il n'y a plus de raison impérieuse pour laquelle chaque hôpital doit avoir son propre processus de délivrance de titres et certificats ou que chaque province doit avoir son propre processus de délivrance de permis d'exercice de la médecine. Il ne fait aucun doute que la simplification de ces processus nous faciliterait la vie, sans compter qu'elle permettrait des économies de coûts pour le système de santé en nous évitant de passer par la filière administrative. Une seule question subsiste: qui en prendra l'initiative?

Intérêts concurrentiels: aucun intérêt déclaré

Mots clés: administration, délivrance de titres et certificats, régimes hospitaliers 